\title{
6 OPEN ACCESS \\ Frameless multimodal image guidance of localized convection-enhanced delivery of therapeutics in the brain
}

ORIGINAL RESEARCH

\author{
Imramsjah M J van der Bom, ${ }^{1}$ Richard P Moser, ${ }^{2}$ Guanping Gao, ${ }^{3}$ \\ Miguel Sena-Esteves, ${ }^{3}$ Neil Aronin, ${ }^{4}$ Matthew J Gounis ${ }^{1}$
}

${ }^{1}$ Department of Radiology, University of Massachusetts, Worcester, Massachusetts, USA

${ }^{2}$ Department of Neurosurgery, University of Massachusetts, Worcester, Massachusetts, USA

${ }^{3}$ Department of Microbiology and Physiological Systems,

Gene Therapy Center, University of Massachusetts, Worcester, Massachusetts, USA

${ }^{4}$ Department of Medicine, Cell Biology, University of Massachusetts, Worcester, Massachusetts, USA

\section{Correspondence to} Dr Matthew Gounis, Department of Radiology, University of Massachusetts, 55 Lake Ave N, SA-107R, Worcester, MA 01655, USA; matthew.gounis@umassmed. edu

Received 21 October 2011 Accepted 22 November 2011 Published Online First 22 December 2011

\begin{abstract}
Introduction Convection-enhanced delivery (CED) has been shown to be an effective method of administering macromolecular compounds into the brain that are unable to cross the blood-brain barrier. Because the administration is highly localized, accurate cannula placement by minimally invasive surgery is an important requisite. This paper reports on the use of an angiographic c-arm system which enables truly frameless multimodal image guidance during CED surgery.

Methods A microcannula was placed into the striatum of five sheep under real-time fluoroscopic guidance using imaging data previously acquired by cone beam computed tomography (CBCT) and MRI, enabling threedimensional navigation. After introduction of the cannula, high resolution CBCT was performed and registered with $\mathrm{MRI}$ to confirm the position of the cannula tip and to make adjustments as necessary. Adeno-associated viral vector-10, designed to deliver small-hairpin micro RNA (shRNAmir), was mixed with $2.0 \mathrm{mM}$ gadolinium (Gd) and infused at a rate of $3 \mu \mathrm{l} / \mathrm{min}$ for a total of $100 \mu \mathrm{l}$. Upon completion, the animals were transferred to an MR scanner to assess the approximate distribution by measuring the volume of spread of $\mathrm{Gd}$.
\end{abstract}

Results The cannula was successfully introduced under multimodal image guidance. High resolution CBCT enabled validation of the cannula position and $\mathrm{Gd}$ enhanced MRI after CED confirmed localized administration of the therapy.

Conclusion A microcannula for CED was introduced into the striatum of five sheep under multimodal image guidance. The non-alloy $300 \mu \mathrm{m}$ diameter cannula tip was well visualized using CBCT, enabling confirmation of the position of the end of the tip in the area of interest.

\section{INTRODUCTION}

Recent developments in genetics and virology have resulted in viral vectors, antibodies and immunotoxins showing promise as therapeutic agents for neurodegenerative disorders such as Huntington's or Parkinson's disease. Although studies have shown their efficacy in preclinical trials, the administration of these new therapeutic agents remains a challenge because their macromolecular size prevents them from crossing the blood-brain barrier, ruling out systemic administration. ${ }^{1} 2$ Intrathecal administration, which is completely driven by free diffusion, leads to non-localized delivery.
In convention-enhanced delivery (CED), therapeutic compounds are forced directly into the region of interest through a needle or cannula by applying a low pressure gradient. ${ }^{3-11}$ A great advantage of CED-based therapy is that it is not limited by the molecular size of the compound, and localized delivery lowers the required volume compared with systemic administration, thus limiting toxicity. Since CED is a localized technique, it is an important requirement that the cannula tip is placed in the target area with a high level of accuracy.

The placement of cannulas, probes and needles is commonly performed by minimally invasive/ stereotactic surgery. These procedures are usually planned using preoperative three-dimensional (3D) patient image data. During the procedure a relationship is established between the image and the patient by a surgical navigation system which enables tracking of surgical tools relative to a fixed reference frame usually attached to the patient. This approach provides high accuracy when a robust relationship between the image and the patient has been established. ${ }^{12} 13$ Although noninvasive methods to obtain this relationship are available, the most reliable methods include fiducial markers or reference frames that are fixed to the skull with surgical screws, which is itself an invasive procedure.

Modern angiography suites are equipped with flat panel c-arm systems that provide two-dimensional (2D) x-ray imaging and also serve as an in situ 3D cone beam CT (CBCT) system. CBCT data are generated by a rotational sweep of the c-arm around the subject, producing a large number of $\mathrm{x}$-ray images. Due to the advancements in flat panel technology and reconstruction software, the image quality is comparable to that of multidetector CT which has enabled the use of numerous applications. ${ }^{14-23}$ Owing to the geometric calibration of the c-arm system, fluoroscopic and in situ acquired CBCT data are coupled, enabling truly frameless navigation. As a result, CBCT data can be used for preoperative planning and for $3 \mathrm{D}$ navigation of the procedure by registration with real-time fluoroscopy. Frameless CBCT guidance with realtime fluoroscopy overlay has been shown to be feasible, safe and reliable for various biopsy and puncture procedures. $^{24-28}$ Although CBCT provides excellent 3D spatial information, it does not provide sufficient soft tissue contrast to enable 
Figure 1 Examples of corresponding coronal slices of $(A)$ T2-weighted turbo spin echo (T2w-TSE), (B) T1-weighted turbo spin echo (T1w-TSE), (C) magnetization prepared rapid gradient echo (MPRAGE) and (D) cone beam CT (CBCT) image data of sheep brain prior to convection-enhanced delivery of therapeutic compound. The burr hole and distal end of the sheath in CBCT data are indicated by the arrow.
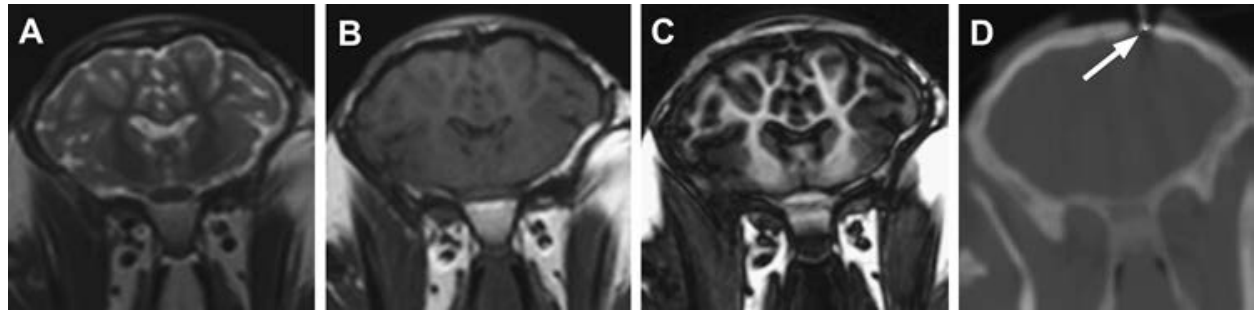

accurate placement of CED devices into a specific region of the brain. MRI accommodates such contrast, and MRI guidance during CED-based surgery would therefore be desirable.

A study was undertaken to establish the feasibility of adenoassociated viral vector (AAV) delivery of small-hairpin micro RNA (shRNAmir) for selective knockdown of mutant huntingtin to slow or block the pathogenesis of Huntington's disease. ${ }^{29}$ To evaluate the safety of the approach and the distribution of AAV-shRNAmir, CED infusions into sheep striatum were performed. In this paper we report on the use of a commercially available truly frameless navigation module (XperGuide, Philips Healthcare, Best, The Netherlands) that enables multimodal image guidance during a CED procedure in a non-invasive manner. Preoperative MRI and CBCT data were registered with real-time fluoroscopy during minimally invasive surgery for CED of this novel therapeutic compound into the striatum of sheep.

\section{MATERIALS AND METHODS}

The experiments were approved by our Institutional Animal Care and Use Committee. As a preclinical trial, surgery was performed in order to perform CED into the striatum of five purposely bred sheep (mean weight $26.5 \mathrm{~kg}$ ). The animals were anesthetized by intramuscular injection of acepromazine $(0.05 \mathrm{mg} / \mathrm{kg})$, buprenorphine $(0.01 \mathrm{mg} / \mathrm{kg})$, glycopyrrolate $(0.01 \mathrm{mg} / \mathrm{kg})$ and thiopental $(15.0 \mathrm{mg} / \mathrm{kg})$ and intravenous injection of ketamine $(3-6 \mathrm{mg} / \mathrm{kg})$ and diazepam $(0.1-0.3 \mathrm{mg} / \mathrm{kg})$. Anesthesia was maintained during the entire procedure. Ventilation was performed mechanically with $2 \%$ isoflurane in oxygen. Physiological monitoring including heart rate, blood pressure, arterial oxygen saturation, temperature, end tidal $\mathrm{CO}_{2}$ and blood gases was performed and recorded every 15 min during the procedure.

Prior to surgery, MRI was performed on a 3 Tesla whole body MRI scanner (Achieva, Philips Healthcare) using an eightchannel knee coil to obtain subject-specific data. Multislice T1and T2-weighted turbo spin echo (TSE) and 3D magnetization prepared rapid gradient echo (MPRAGE) images were acquired with a field of view of $128 \times 128 \times 80 \mathrm{~mm}^{3}$, an in-plane resolution of $1 \times 1 \mathrm{~mm}$ and slice thickness of $2 \mathrm{~mm}$. The total imaging time was approximately $25 \mathrm{~min}$. Upon completion, the animals were transferred to the adjacent angiography suite and prepared for sterile surgery. They were secured on the non-radio-opaque table with a surgical beanbag. A non-invasive frame designed to hold and manipulate the access sheath was mounted onto the animal's skull. A single incision and burr hole $(0.5 \mathrm{~cm}$ diameter $)$ were created approximately $1 \mathrm{~cm}$ anterior of the coronal suture and $1 \mathrm{~cm}$ mediolateral to the sagittal suture. A metal sheath was inserted into the manipulator with the distal end touching the dura mater. CBCT data were acquired for surgery planning and navigation using an angiographic c-arm system (Allura FD20, Philips Healthcare). Preoperative MRI data were imported into the corresponding work station and registered with CBCT data. The path of the CED microcannula was planned on merged datasets using XperGuide. This dedicated navigation software provides real-time guidance of radio-dense medical devices by fluoroscopic imaging shown relative to $3 \mathrm{D}$ multimodal image
Figure 2 Snapshots of navigation module used for surgical navigation during convection-enhanced delivery. Real-time $x$-ray images (gray scale) are superimposed onto cone beam CT (CBCT) (red scale) and MRI (blue scale) data. (A) The entry point, target point and planned path are indicated by the pink and green circles and green dots, respectively. After each alteration of the position and orientation of the sheath the x-ray image was updated. Optimal alignment was achieved by alternating the orientation between the entry view $(B, C)$ and progression view $(D, E)$.
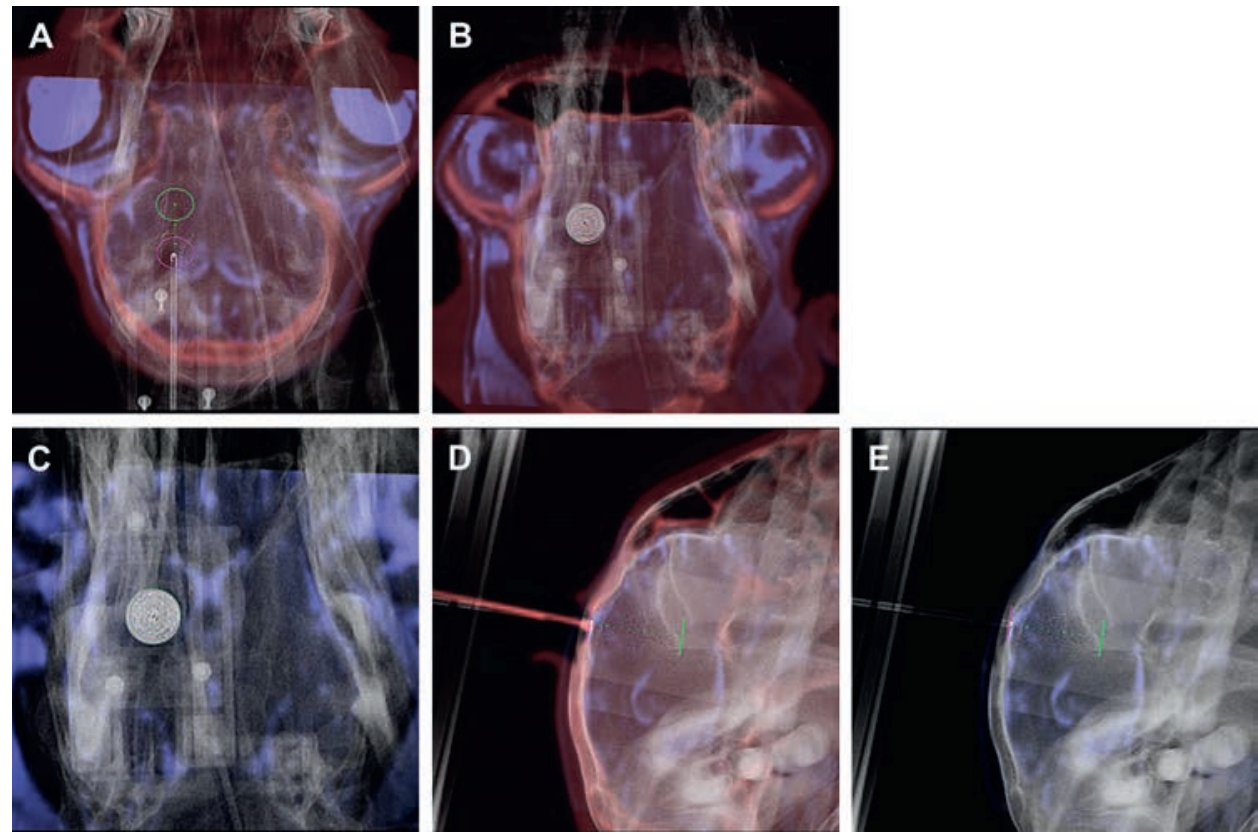
Figure 3 (A) Scale image of the distal end of the microcannula. The cannula tip (arrows) has a length of $3 \mathrm{~mm}$ and a diameter of $300 \mu \mathrm{m}$. (B) Maximum intensity projection of a $0.2 \mathrm{~mm}$ slab of a high resolution cone beam CT (CBCT) (red scale) acquired after introduction of the convection-enhanced delivery cannula. The small detector size and unbinned reconstruction algorithm enables in vivo visualization of the complete cannula. (C) CBCT was registered with preoperative T2-weighted turbo spin echo (gray scale) to confirm the end position and make adjustments in the direction of progression if necessary.

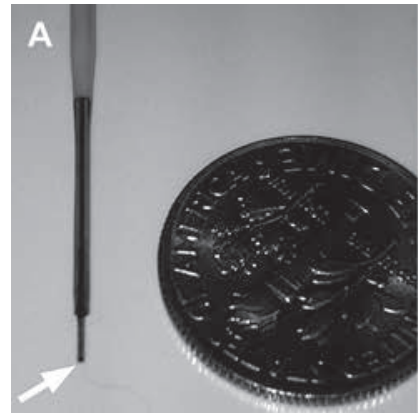

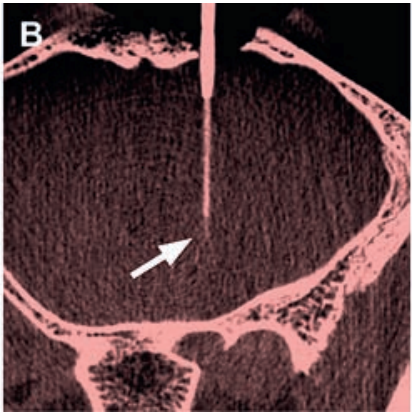

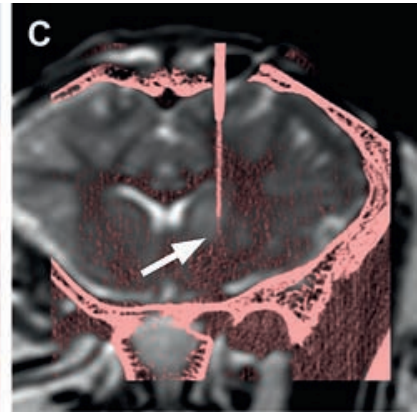

data and the planned path in a single view. Fluoroscopic data were updated in conjunction with every adjustment of the manipulator in order to see the effects in relation to 3D image data and the planned path. By alternating between the entry view (ie, parallel to the planned path) and progression view (ie, perpendicular to the planned path), both automatically calculated and provided by navigation software, the sheaths were readily aligned with the planned paths. The cannula with a distal tip length of $3.0 \mathrm{~mm}$ and outer diameter of $300 \mu \mathrm{m}$ was introduced and progressed towards the end position without fluoroscopic guidance because the cannula tip was not visible with x-ray imaging. Instead, progression depth measured from the dura mater during the planning phase was applied. The end position of the cannula tip was validated by acquisition of high resolution CBCT data (XperCT) using a $22 \mathrm{~cm}$ detector size and an unbinned reconstruction algorithm. ${ }^{19}$ High resolution CBCT was registered to T2w-TSE data to confirm the position of the cannula tip and, if necessary, the cannula was progressed by distance to the target measured on the fused image data.

In order to visualize the approximate spread of the injected substance with MRI, the therapeutic compound was mixed with $2.0 \mathrm{mmol} / \mathrm{l}$ gadolinium (Gd). The mixture was infused at a rate of $0.3 \mu \mathrm{l} / \mathrm{min}$ for a total of $100 \mu \mathrm{l}$ using a syringe pump (PHD 2000 Infusion, Harvard Apparatus, Holliston, Massachusetts, USA). After infusion, the skull and wound were closed and the animal was transferred to the MRI system where the same image data were acquired as before surgery. Upon completion, the animals were recovered.

\section{RESULTS}

CED surgery was planned on preoperatively acquired MRI (figure $1 \mathrm{~A}-\mathrm{C}$ ) and CBCT data obtained after creation of the burr hole (figure 1D). Cannulae were successfully introduced under multimodal image guidance. Figure 2 shows snapshots of fluo- roscopic data (gray scale) overlaid with CBCT data (red scale) and MRI data (blue scale). The cannula tip, of outer diameter $300 \mu \mathrm{m}$ (figure $3 \mathrm{~A}$ ), was visualized in vivo using high resolution CBCT (figure $3 \mathrm{~B}$ ), and registration with MRI enabled validation of the position relative to the striatum (figure 3C). Gd-enhanced MRI after CED confirmed the localized administration of the compound and was used to measure the approximate distribution volume (figure 4).

\section{DISCUSSION}

CED has been shown to be an effective method of administering macromolecular drugs into the brain. However, accurate placement of a delivery device remains a challenge that requires excellent surgical skills. Advancements in imaging and navigation technology may be of great value in assisting the surgeon during this procedure. In this short report, we demonstrate the use of a commercially available truly frameless navigation module that provides multimodal image guidance during surgery for CED. Although neither a quantitative analysis nor a comparison with other navigation techniques was performed, we have shown that the XperGuide module can be of value for $\mathrm{CED}$ and other procedures that may require accurate positioning such as biopsy, ablation and stimulation.

A great advantage of frameless navigation is that it does not require the use of invasive fiducials or additional navigation machinery. Due to calibration of the c-arm system, the relationship between the image and the patient is simply established by in situ acquisition of 3D image data which can subsequently be used for navigation or merged with other patient data.

With high resolution CBCT we were able to image the small distal end of the microcannula in vivo. Using MRI data, this enabled us to visualize the exact end position of the cannula tip and to make adjustments to the depth as necessary. This represents a significant advantage over other surgical navigation
Figure 4 Examples of corresponding coronal slices of (A) T2-weighted turbo spin echo (T2w-TSE), (B) T1-weighted turbo spin echo (T1w-TSE) and (C) magnetization prepared rapid gradient echo (MPRAGE) image data of sheep brain after convection-enhanced delivery of therapeutic compound mixed with gadolinium. The spread of the therapeutic compound is assumed to be similar to that of gadolinium, which is clearly visible on both $\mathrm{T} 1$-weighted sequences (arrows).

systems in that direct rather than inferred confirmation of the cannula position can be obtained.

A limitation of using truly frameless navigation is that it requires the acquisition of CBCT data and real-time fluoroscopy, which exposes the patient to additional ionizing radiation. The acquisition of a single CBCT dataset produces a radiation dose of approximately $50 \mathrm{mGy}$, which can be lowered by using a lowdose CBCT protocol, although at the cost of signal-to-noise. Another limitation of the navigation method used is that a relationship between the patient and the image will only be maintained provided the patient does not move during the procedure. Although extensive research has been performed to realign patient and volumetric data using fluoroscopic images when the patient has moved, ${ }^{30}$ currently a relationship can only be re-established by acquiring new CBCT data.

\section{CONCLUSIONS}

State-of-the-art angiographic c-arm systems contain hardware and software technology that enable truly frameless multimodal image guidance which may be of great value during minimally invasive procedures.

Acknowledgments The authors acknowledge Philips Healthcare and Lundbeck Inc.

Funding This research was funded by a grant from Lundbeck Inc.

Competing interests NA is a member of the NIH sponsored DERC (DK 032520).

Contributors IMJvdB: study design, data collection, data analysis, manuscript preparation. RPM: study design, operating neurosurgeon, manuscript editing. GG: study design, material production, manuscript editing. MS-E: study design, manuscript editing. NA: PI. MG: study design, data analysis, manuscript preparation.

Provenance and peer review Not commissioned; externally peer reviewed.

Open Access This is an Open Access article distributed in accordance with the Creative Commons Attribution Non Commercial (CC BY-NC 3.0) license, which permits others to distribute, remix, adapt, build upon this work non-commercially, and license their derivative works on different terms, provided the original work is properly cited and the use is non-commercial. See: http://creativecommons.org/ licenses/by-nc/3.0/

\section{REFERENCES}

1. Sah DW, Aronin N. Oligonucleotide therapeutic approaches for Huntington disease. J Clin Invest 2011:121:500-7.

2. Bankiewicz KS, Leff SE, Nagy D, et al. Practical aspects of the development of ex vivo and in vivo gene therapy for Parkinson's disease. Exp Neurol 1997;144:147-56.

3. Lonser RR, Walbridge S, Garmestani K, et al. Successful and safe perfusion of the primate brainstem: in vivo magnetic resonance imaging of macromolecular distribution during infusion. J Neurosurg 2002;97:905-13.

4. Szerlip NJ, Walbridge S, Yang L, et al. Real-time imaging of convection-enhanced delivery of viruses and virus-sized particles. J Neurosurg 2007;107:560-7.

5. Voges J, Reszka R, Gossmann A, et al. Imaging guided convection enhanced delivery and gene therapy of glioblastoma. Ann Neurol 2003;54:479-87.

6. Lonser RR, Schiffman R, Robison RA, et al. Image-guided, direct convective delivery of glucocerebrosidase for neuronopathic Gaucher disease. Neurology 2007:68:254-61.

7. Heiss JD, Walbridge $S$, Asthagiri AR, et al. Image-guided convection-enhanced delivery of muscimol to the primate brain. J Neurosurg 2010;112:790-5.
8. Murad GJA, Walbridge S, Morrison PF, et al. Image-guided convection-enhanced delivery of gemcitabine to the brainstem. J Neurosurg 2007;106:351-6.

9. Bankiewicz KS, Eberling JL, Kohutnicka M, et al. Convection-enhanced delivery of AAV vector in parkinsonian monkeys; in vivo detection of gene expression and restoration of dopaminergic function using pro-drug approach. Exp Neurol 2000:164:2-14.

10. Song DK, Lonser RR. Convection-enhanced delivery for the treatment of pediatric neurologic disorders. J Child Neurol 2008;23:1231-7.

11. Richardson RM, Kells AP, Martin AJ, et al. Novel platform for MRI-guided convection-enhanced delivery of therapeutics: preclinical validation in nonhuman primate brain. Stereotact Funct Neurosurg 2011;89:141-51.

12. Brown RA. A computerized tomography-computer graphics approach to stereotaxic localization. J Neurosurg 1979;50:715-20.

13. Peters TM. Image-guidance for surgical procedures. Phys Med Biol 2006:51:R505.

14. Struffert T, Richter G, Engelhorn T, et al. Visualisation of intracerebral haemorrhage with flat-detector CT compared to multislice CT: results in 44 cases. Eur Radiol 2009;19:619-25

15. Heran NS, Song JK, Namba K, et al. The utility of DynaCT in neuroendovascular procedures. AJNR Am J Neuroradiol 2006;27:330.

16. van der Bom IMJ, Mehra M, Walvick RP, et al. Quantitative evaluation of c-arm CT CBV in a canine model of ischemic stroke. AJNR Am J Neuroradiol 2011. Published Online First: 15 December 2011. doi:10.3174/ajnr.A2944.

17. Doelken M, Struffert T, Richter G, et al. Flat-panel detector volumetric CT for visualization of subarachnoid hemorrhage and ventricles: preliminary results compared to conventional CT. Neuroradiology 2008;50:517-23.

18. Struffert T, Deuerling-Zheng Y, Kloska S, et al. Flat detector CT in the evaluation of brain parenchyma, intracranial vasculature, and cerebral blood volume: a pilot study in patients with acute symptoms of cerebral ischemia. AJNR Am J Neuroradiol 2010;31:1462-9

19. Patel NV, Gounis MJ, Wakhloo AK, et al. Contrast-enhanced angiographic cone-beam CT of cerebrovascular stents: experimental optimization and clinical application. AJNR Am J Neuroradiol 2011;32:137.

20. Ganguly A, Fieselmann A, Marks M, et al. Cerebral CT perfusion using an interventional c-arm imaging system: cerebral blood flow measurements. AJNR Am J Neuroradiol 2011;32:1525-31.

21. Ahmed AS, Zellerhoff M, Strother CM, et al. C-arm CT measurement of cerebral blood volume: an experimental study in canines. AJNR Am J Neuroradiol 2009:30:917-22.

22. Bley T, Strother CM, Pulfer K, et al. C-Arm CT measurement of cerebral blood volume in ischemic stroke: an experimental study in canines. AJNR Am J Neuroradio 2010:31:536-40.

23. Söderman $\mathbf{M}$, Babic D, Holmin $\mathbf{S}$, et al. Brain imaging with a flat detector $\mathbf{C}$-arm. Neuroradiology 2008;50:863-8.

24. Racadio JM, Babic D, Homan R, et al. Live 3D guidance in the interventional radiology suite. Am J Roentgenol 2007;189:W357-64.

25. Kroeze SG, Huisman M, Verkooijen HM, et al. Real-time 3D fluoroscopy-guided large core needle biopsy of renal masses: a critical early evaluation according to the IDEAL recommendations. Cardiovasc Intervent Radiol. Published Online First: 6 August 2011. doi:10.1007/s00270-011-0237-4.

26. Braak SJ, van Strijen MJL, van Leersum M, et al. Real-time 3D fluoroscopy guidance during needle interventions: technique, accuracy, and feasibility. Am J Roentgenol 2010;194:W445-51.

27. Leschka SC, Babic D, El Shikh S, et al. C-arm cone beam computed tomography needle path overlay for image-guided procedures of the spine and pelvis. Neuroradiology. Published Online First: 8 April 2011. doi:10.1007/s00234-011-0866-y.

28. Heye S, Vaninbroukx J, Daenens K, et al. Embolization of an internal iliac artery aneurysm after image-guided direct puncture. Cardiovasc Intervent Radiol. Published Online First: 18 December 2010. doi:10.1007/s00270-010-0061-2.

29. Pfister EL, Kennington L, Straubhaar J, et al. Five siRNAs targeting three SNPs may provide therapy for three-quarters of Huntington's disease patients. Curr Biol 2009;19:774-8.

30. Markelj P, Tomazevic D, Likar B, et al. A review of 3D/2D registration methods for image-guided interventions. Med Image Anal. Published Online First: 13 April 2010. doi:10.1016/j.media.2010.03.005 